\title{
Adaptive Fault-Tolerant Control for Flight Systems with Input Saturation and Model Mismatch
}

\author{
Man Wang, ${ }^{1}$ Jianying Yang, ${ }^{1}$ and ${\mathrm{Nan} \mathrm{Li}^{2}}^{2}$ \\ ${ }^{1}$ Department of Mechanics and Aerospace Engineering, State Key Laboratory for Turbulence and Complex Systems, \\ College of Engineering, Peking University, Beijing 100871, China \\ ${ }^{2}$ School of Automation, Chongqing University, Chongqing 400044, China
}

Correspondence should be addressed to Jianying Yang; yjy@pku.edu.cn

Received 1 August 2013; Accepted 12 August 2013

Academic Editor: Guanghui Wen

Copyright (c) 2013 Man Wang et al. This is an open access article distributed under the Creative Commons Attribution License, which permits unrestricted use, distribution, and reproduction in any medium, provided the original work is properly cited.

\begin{abstract}
A novel scheme for fault-tolerant control is proposed in this paper, in which model reference adaptive control method is incorporated with control allocation to cope with simultaneous actuator failures, input saturation, and model mismatch in the flight system. In order to reduce performance degradation caused by actuator failures, the proposed scheme redistributes the control signal to healthy actuators and updates the weighting matrix based on actuator effectiveness. Because of saturation errors resulting from actuator constraints and model mismatch caused by abnormal changes in the system, the original reference model may not be appropriate. Under this circumstance, an adaptive reference model which can also provide satisfactory performance is designed. Simulations of a flight control example are given to illustrate the effectiveness of the proposed scheme.
\end{abstract}

\section{Introduction}

Actuator failures may adversely affect the stability and performance of flight systems. Fault-tolerant control (FTC) has been studied as a method for accommodating failures. In the past decades, a number of approaches have been proposed for FTC, including sliding mode control-based designs $[1,2]$, variable structure control-based designs [3], learning-based approaches [4], and adaptive control-based designs [5].

The availability of actuator redundancy is an important element of FTC. Control allocation (CA) is an approach that can effectively manage redundancy by distributing virtual control law requirements to redundant actuators in the most efficient manner [6]. There is an extensive literature on CA approaches and applications. Enns presented the linear and quadratic programming approaches in detail in [7]. Härkegård and Glad compared control allocation with optimal control for solving actuator redundancy in [8]. Several CA approaches taking into account actuator limits have been discussed [9-11]. Kishore et al. gave an algorithm that updated the weighting matrix to deal with actuator limits in [9]. However, in the event of failure, only depending on updating the weighting matrix sometimes may be invalid.

In addition, flight systems may experience other disruptive factors such as model mismatch. Model mismatch can be caused by icing or damage to the aircraft. Joshi et al. first considered this problem in [12] and further developed his work in [13]. Although the previous work has achieved satisfactory tracking performance, none of them considered actuator limits.

In this paper, we develop a novel scheme combining CA with model reference adaptive control (MRAC) for flight systems with simultaneous actuator failures, model mismatch, and input saturation. On the basis of the existing literature, this paper proposes an improved control allocation (ICA) method that updates the weighting matrix based on actuator effectiveness. In addition to updating the weighting matrix, this paper employs the positive $\mu$-modification method [14] to impose constraints on actuators. MRAC can guarantee the closed-loop stability of the system and reduce the error caused by actuator failures. Because of input saturation and model mismatch, the original reference model may not 
be appropriate. We have designed an adaptive reference model that can provide satisfactory performance under these circumstances. Compared with the traditional CA methods, simulation results show that the new scheme provides better tracking performance. The control structure is shown in Figure 1. This paper focuses on the tracking control on the single system. In the recent study, the coordination control of multiagent systems has attracted considerable attention. Several approaches and applications have been developed in [15-17]. We will explore the FTC of multiple flight systems in our future study.

This paper is organized as follows. Section 2 presents the problem statement. Section 3 gives a detailed description of the novel adaptive fault-tolerant control scheme. Simulation results of a flight control example are given in Section 4. Section 5 is concluding remarks.

\section{Problem Statement}

Consider a linear time-invariant system in the presence of model mismatch and actuator failures described by

$$
\begin{gathered}
\dot{x}(t)=\left(A+\delta_{a}\right) x(t)+B_{u} \Delta u(t), \\
y(t)=C x(t),
\end{gathered}
$$

where $x(t) \in \mathfrak{R}^{n}$ is the state vector, $u(t) \in \mathfrak{R}^{m}$ is the actual control input, $y(t) \in \Re^{p}$ is the output, and $A, B_{u}, C$ are constant matrices of appropriate dimensions. The matrix $\delta_{a} \in \mathfrak{R}^{n \times n}$ denotes unknown parameter deviations or model mismatch caused by icing, modeling errors, or damage. The actuator effectiveness matrix $\Delta \in \mathfrak{R}^{m \times m}$ is a piecewise constant uncertain matrix which can be expressed as $\Delta=$ $\operatorname{diag}\left(\delta_{1}, \delta_{2}, \ldots, \delta_{m}\right)$, where $0 \leq \delta_{i} \leq 1, i=1,2, \ldots, m$, indicates the effectiveness of the $i$ th actuator. For example, $\delta_{i}=1$ indicates that the $i$ th actuator is working well; $\delta_{i}=0$ implies that the $i$ th actuator has damaged completely; $0<$ $\delta_{i}<1$ means that the $i$ th actuator has partially lost its effectiveness.

Assumption 1. Each element of the control input $u(t)$ is bounded by a constant such that

$$
\left|u_{i}\right| \leq u_{\max _{i}} \quad \forall t>0, i=1,2, \ldots, m .
$$

Assumption 2. There is sufficient actuator redundancy in the system so that the input matrix $B_{u}$ can be factorized into a full column rank matrix $B_{v} \in \Re^{n \times p}$ and a full row rank matrix $B \in \mathfrak{R}^{p \times m}$; that is,

$$
B_{u}=B_{v} B
$$

Assumption 3. $\left(A, B_{u} \Delta\right)$ is controllable.

Assumption 4. For the nominal system with $\Delta=I$ and $\delta_{a}=0$, there exist gains $K_{1} \in \mathfrak{R}^{n \times p}$ and $K_{2} \in \mathfrak{R}^{p \times p}$ satisfying the following matching conditions:

$$
A+B_{v} K_{1}^{T}=A_{m} ; \quad B_{v} K_{2}=B_{m}
$$

where $A_{m} \in \mathfrak{R}^{n \times n}$ is Hurwitz and $B_{m} \in \mathfrak{R}^{n \times p}$.

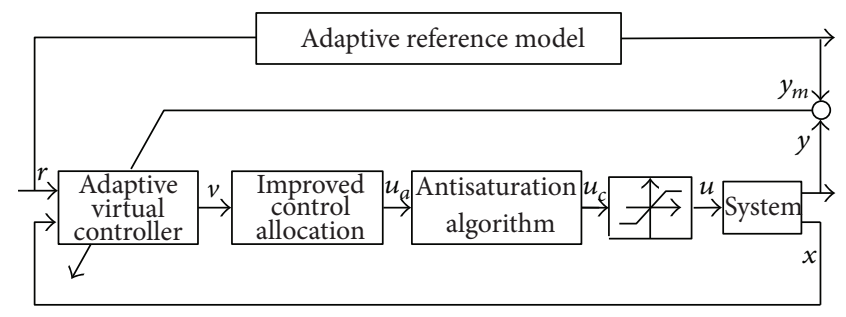

FIGURE 1: The overall control structure.

Based on Assumption 1, the static actuator model can be defined as follows [14]:

$$
\begin{aligned}
u_{i}(t) & =u_{\max _{i}} \operatorname{sat}\left(\frac{u_{c_{i}}(t)}{u_{\max _{i}}}\right) \\
& = \begin{cases}u_{c_{i}}(t), & \left|u_{c_{i}}(t)\right| \leq u_{\text {max }_{i}}, \quad i=1, \ldots, m, \\
u_{\max _{i}} \operatorname{sgn}\left(u_{c_{i}}(t)\right), & \left|u_{c_{i}}(t)\right|>u_{\text {max }_{i}},\end{cases}
\end{aligned}
$$

where $u_{c_{i}}(t)$ denotes the $i$ th element of the commanded control input $u_{c}(t)$. Based on the positive $\mu$-modification method [14], the commanded control input $u_{c}(t)$ can be obtained by

$$
u_{c}(t)=u_{a}(t)+\mu \tilde{u}_{c}(t)
$$

where $\mu \in \mathfrak{R}^{m \times m}$ is a diagonal matrix of design constants $\mu_{1}, \ldots, \mu_{m} \geq 0, u_{a}(t)$ is the adaptive control input, and $\tilde{u}_{c}(t)=$ $\left[\tilde{u}_{c_{1}}(t), \ldots, \tilde{u}_{c_{m}}(t)\right]^{T}, \tilde{u}_{c_{i}}(t)=u_{\max _{i}}^{\delta_{i}} \operatorname{sat}\left(u_{c_{i}}(t) / u_{\text {max }_{i}}^{\delta_{i}}\right)-u_{c_{i}}(t), i=$ $1, \ldots, m$.

In addition, the saturation error can be defined as

$$
\widetilde{u}(t)=u(t)-u_{c}(t)
$$

which may lead to performance degradation or even system instability.

Based on Assumption 4, we can define the conventional reference model as

$$
\dot{x}_{m}(t)=A_{m} x_{m}(t)+B_{m} r(t)
$$

where $x_{m}(t) \in \mathfrak{R}^{n}$ is the reference model state and $r(t) \in \mathfrak{R}^{p}$ is a bounded reference input. Optimal and robust control theory can be employed to design the gain matrices $K_{1}, K_{2}$. However, due to model mismatch $\delta_{a},\left(A+\delta_{a}+B_{v} K_{1}^{T}\right)$ will not have the same structure as $A_{m}$ for any $K_{1}$. Therefore, stability and asymptotic tracking performance can no longer be guaranteed.

In response, this paper designs an adaptive reference model while taking saturation error $\tilde{u}$ and model mismatch $\delta_{a}$ into consideration. The control objective is to develop a novel adaptive fault-tolerant control scheme, for the faulty system given by (1) with simultaneous actuator failures, model mismatch, and input saturation, to achieve the desired tracking performance. 


\section{Adaptive Fault-Tolerant Control}

In order to achieve the desired control objective, we propose an adaptive fault-tolerant control scheme in this section. The scheme mainly incorporates an improved control allocation method and an adaptive virtual controller design.

3.1. Improved Control Allocation Method. Substituting (6) and (7) into (1) yields the following closed-loop system dynamics:

$$
\dot{x}(t)=\left(A+\delta_{a}\right) x(t)+B_{f} u_{a}(t)+B_{f} \widetilde{u}_{a}(t),
$$

where

$$
B_{f}=B_{u} \Delta \widetilde{u}_{a}(t)=\mu \widetilde{u}_{c}(t)+\widetilde{u}(t) .
$$

Based on Assumption 2, we can get

$$
B_{f} u_{a}(t)=B_{v} B \Delta u_{a}(t)=B_{v} v(t)
$$

where $v(t) \in \mathfrak{R}^{p}$ is a virtual control input that represents the total control effort produced by actuators.

As distinct from conventional CA problem, the matrix $B_{f}$ incorporates fault information. Generally, CA can be formulated as an optimization problem as follows [1]:

$$
\min _{u_{a}(t)} u_{a}^{T} W u_{a}, \quad \text { subject to }(11)
$$

where $W=\operatorname{diag}\left(w_{1}, w_{2}, \ldots, w_{m}\right)$ is a positive definite weighting matrix and the scalar $w_{i}$ represents the weight of the $i$ th actuator.

In contrast to the preceding CA results, the weighting matrix $W$ can be updated online based on actuator effectiveness in this paper. Suppose that the $i$ th actuator has partially lost effectiveness; that is, $\Delta=\operatorname{diag}\left(1, \ldots, 1, \delta_{i}, 1, \ldots, 1\right), 0<$ $\delta_{i}<1$. Let the $i$ th diagonal element of $W$ be perturbed from $w_{i}$ to $\kappa_{i}^{2} w_{i}$, where the scalar $\kappa_{i}$ can be obtained by $\kappa_{i}=1 / \delta_{i}$. Then the new weighting matrix becomes $\widehat{W}=\vartheta W \vartheta$, where $\vartheta=\operatorname{diag}\left(1, \ldots, 1, \kappa_{i}, 1, \ldots, 1\right)$. Consequently, the solution to (11) can be formulated as

$$
u_{a}(t)=\widehat{W}^{-1} B_{f}^{T}\left(B_{f} \widehat{W}^{-1} B_{f}^{T}\right)^{-1} v(t)
$$

Usually the initial value of the weighting matrix $W$ is selected based on the priority of actuators. Once actuator failures occur, $W$ will be updated. As $\delta_{i} \rightarrow 0, \kappa_{i} \rightarrow \infty$, the associated component $u_{i}$ in (12) is therefore weighted heavily since $w_{i}$ becomes large. If the $i$ th actuator has damaged completely, the value of $w_{i}$ will be infinity. Equation (13) is able to distribute the virtual control signal to the remaining physical actuators based on actuator effectiveness. However, due to the failure, only depending on the adaption of $W$ to avoid saturation sometimes may be invalid. Therefore, this paper employs a positive $\mu$-modification method to ensure that all control inputs lie within the bounds.

The following lemma gives the algorithm of the positive $\mu$-modification method.
Lemma 5 (see [14]). If $\mu_{1}, \ldots, \mu_{m} \geq 0$, then the solution to (6) is given by a convex combination of $u_{a}(t)$ and $u_{\max }^{\delta} \operatorname{sat}\left(u_{a}(t) / u_{\max }^{\delta}\right)$ for all $t>0$ :

$$
u_{c}(t)=\left(I_{q}+\mu\right)^{-1}\left(u_{a}(t)+\mu u_{\max }^{\delta} \operatorname{sat}\left(u_{\max }^{\delta}{ }^{-1} u_{a}(t)\right)\right)
$$

which can also be represented component-wise in the following form:

$$
\begin{aligned}
& u_{c_{i}}(t) \\
& = \begin{cases}u_{a_{i}}(t), & \left|u_{a_{i}}(t)\right| \leq u_{\text {max }_{i}}^{\delta_{i}}, \\
\frac{1}{1+\mu_{i}}\left(u_{a_{i}}(t)+\mu_{i} u_{\max _{i}}^{\delta_{i}}\right), & u_{a_{i}}(t)>u_{\max _{i}}^{\delta_{i}}, \quad i=1, \ldots, m . \\
\frac{1}{1+\mu_{i}}\left(u_{a_{i}}(t)-\mu_{i} u_{\max _{i}}^{\delta_{i}}\right), & u_{a_{i}}(t)<-u_{\max _{i}}^{\delta_{i}},\end{cases}
\end{aligned}
$$

Remark 6. Detailed discussion of the above lemma can be found in [14].

3.2. Adaptive Virtual Controller Design. The adaptive controller is designed as

$$
v(t)=\widehat{K}_{1} x(t)+\widehat{K}_{2} r(t),
$$

where $\widehat{K}_{1}$ and $\widehat{K}_{2}$ are the estimates of $K_{1}$ and $K_{2}$, respectively.

Substituting (11) and (16) into (9) yields the closed-loop system

$$
\begin{aligned}
\dot{x}= & \left(A+B_{v} K_{1}+\delta_{a}\right) x+B_{v} K_{2} r \\
& +B_{v} \widetilde{K}_{1} x+B_{v} \widetilde{K}_{2} x+B_{v} B_{f} \widetilde{u}_{a},
\end{aligned}
$$

where $\widetilde{K}_{1}=\widehat{K}_{1}-K_{1}, \widetilde{K}_{2}=\widehat{K}_{2}-K_{2}$.

In order to compensate for the saturation error $\widetilde{u}_{a}$, we design an adaptive compensation controller $\widehat{K}_{3} \in \mathfrak{R}^{p \times p}$. The initial value of $\widehat{K}_{3}$ is defined as $K_{3}$ and satisfies

$$
K_{2} K_{3}=I \text {. }
$$

Using (4) and (18), the closed-loop system in (17) can be modified as

$$
\dot{x}=\left(A_{m}+\delta_{a}\right) x+B_{m}\left(r+K_{3} B_{f} \widetilde{u}_{a}\right)+B_{v} \widetilde{K}_{1} x+B_{v} \widetilde{K}_{2} r .
$$

The modified closed-loop system dynamics lead to consideration of the following adaptive reference model system:

$$
\dot{x}_{m}=A_{M} x_{m}+B_{m}\left(r+\widehat{K}_{3} B_{f} \tilde{u}_{a}\right),
$$

where $A_{M}$ is a time-varying matrix incorporating the model mismatch estimate $\widehat{\delta}_{a}$, denoted as

$$
A_{M}=A_{m}+\widehat{\delta}_{a} .
$$

Following [18], the system matrix $A_{M}$ can be described as an affine function of a parameter vector $\rho \in R^{n_{\rho}}$ that lies in a convex polytope $\mathcal{S}$ having vertices $\rho^{j}, j=1,2, \ldots n_{v}$; that is,

$$
A_{M}[\rho(t)]=A_{m}+\sum_{1}^{n_{\rho}} \rho_{i}(t) A_{M_{i}},
$$


where $A_{M_{i}}$ are constant matrices and $\rho_{i}(t) \in\left[\rho_{i_{\min }}, \rho_{i_{\max }}\right]$. Suppose that $A_{M}\left(\rho^{j}\right)$ denotes the value of the reference model system matrix at vertex $\rho^{j}$ and there exist positive definite matrices $P=P^{T}, Q=Q^{T} \in \mathfrak{R}^{n \times n}$ satisfying

$$
A_{M}\left(\rho^{j}\right)^{T} P+P A_{M}\left(\rho^{j}\right)<-Q, \quad j=1, \ldots, n_{v} .
$$

Based on (23), we can get the following Lyapunov inequality for all $\rho(t) \in \mathcal{S}$ :

$$
A_{M}[\rho(t)]^{T} P+P A_{M}[\rho(t)]<-Q, \quad j=1, \ldots, n_{v} .
$$

Thus the autonomous part of the adaptive reference model in (20) is exponentially stable for all $\rho(t) \in \mathcal{S}$ [18].

Note that this paper only considers the case that the true value of model mismatch lies within the stable region Figure 6 . The model mismatch $\delta_{a}$ is assumed to satisfy

$$
\left(A_{m}+\delta_{a}\right)^{T} P+P\left(A_{m}+\delta_{a}\right)<-Q .
$$

Subtracting (20) from (19) yields the following error dynamics:

$$
\dot{e}=A_{M} e+B_{v} \widetilde{K}_{1} x+B_{v} \widetilde{K}_{2} r-\widetilde{\delta}_{a} x-B_{m} \widetilde{K}_{3} B_{f} \widetilde{u}_{a},
$$

where $e=x-x_{m}$ and $\widetilde{\delta}_{a}=\widehat{\delta}_{a}-\delta_{a}$ denote the tracking error and model mismatch estimate error, respectively.

The following theorem gives the adaption and parameter estimation laws which ensure that $e(t) \rightarrow 0$ and all signals remain bounded. We will first assume that the initial conditions satisfy Theorem 4.1 in [14].

Theorem 7. For the system given by (1), the adaptive controller (16) with gain adaption laws

$$
\begin{gathered}
\dot{\widehat{K}}_{1}=-\Gamma_{1} B_{v}^{T} \operatorname{Pex}^{T}, \\
\dot{\widehat{K}}_{2}=-\Gamma_{2} B_{v}^{T} \operatorname{Per}^{T}
\end{gathered}
$$

and parameter estimation laws

$$
\begin{gathered}
\dot{\widehat{K}}_{3}=\Gamma_{3} B_{m}^{T} P e\left(B_{f} \tilde{u}_{a}\right)^{T}, \\
\dot{\widehat{\delta}}_{a}=\Gamma_{a} P e x^{T},
\end{gathered}
$$

where $\Gamma_{1}, \Gamma_{2}, \Gamma_{3} \in \mathfrak{R}^{p \times p}$ and $\Gamma_{a} \in \mathfrak{R}^{n \times n}$ are positive definite matrices and $P$ is determined by (24), guarantees that all the closed-loop signals remain bounded and that the tracking error $e(t)$ converges to 0 as $t \rightarrow \infty$.

Proof. In order to analyze the closed-loop stability and tracking performance, choose a Lyapunov function as

$$
\begin{aligned}
V= & e^{T} P e+\sum_{i=1}^{m} \widetilde{\delta}_{a i}^{T} \Gamma_{a}^{-1} \widetilde{\delta}_{a i}+\operatorname{tr}\left(\widetilde{K}_{1}^{T} \Gamma_{1}^{-1} \widetilde{K}_{1}\right) \\
& +\operatorname{tr}\left(\widetilde{K}_{2}^{T} \Gamma_{2}^{-1} \widetilde{K}_{2}\right)+\operatorname{tr}\left(\widetilde{K}_{3}^{T} \Gamma_{3}^{-1} \widetilde{K}_{3}\right)
\end{aligned}
$$

where $\operatorname{tr}(\cdot)$ denotes the trace of a matrix and $\widetilde{\delta}_{a i}$ denotes the $i$ th column of $\widetilde{\delta}_{a}$.

Differentiating (29), we can get

$$
\begin{aligned}
\dot{V}= & 2 e^{T} P\left(A_{M} e+B_{v} \widetilde{K}_{1} x+B_{v} \widetilde{K}_{2} r-\widetilde{\delta}_{a} x-B_{m} \widetilde{K}_{3} B_{f} \widetilde{u}_{a}\right) \\
& +\sum_{i=1}^{m} \widetilde{\delta}_{a i}^{T} \Gamma_{a}^{-1} \widetilde{\delta}_{a i}+\operatorname{tr}\left(\widetilde{K}_{1}^{T} \Gamma_{1}^{-1} \dot{\widetilde{K}}_{1}\right) \\
& +\operatorname{tr}\left(\widetilde{K}_{2}^{T} \Gamma_{2}^{-1} \dot{\widetilde{K}}_{2}\right)+\operatorname{tr}\left(\widetilde{K}_{3}^{T} \Gamma_{3}^{-1} \dot{\widetilde{K}}_{3}\right) \\
< & -e^{T} Q e-2 \operatorname{tr}\left[\widetilde{\delta}_{a}^{T}\left(P e x^{T}-\Gamma_{a}^{-1} \dot{\widehat{\delta}}_{a}\right)\right] \\
& +2 \operatorname{tr}\left[\widetilde{K}_{1}^{T}\left(B_{v}^{T} P e x^{T}+\Gamma_{1}^{-1} \dot{\widetilde{K}}_{1}\right)\right] \\
& +2 \operatorname{tr}\left[\widetilde{K}_{2}^{T}\left(B_{v}^{T} P e r{ }^{T}+\Gamma_{2}^{-1} \dot{\widetilde{K}}_{2}\right)\right] \\
& -2 \operatorname{tr}\left[\widetilde{K}_{3}^{T}\left(B_{m}^{T} P e\left(B_{f} \widetilde{u}\right)^{T}-\Gamma_{3}^{-1} \dot{\widetilde{K}}_{3}\right)\right] .
\end{aligned}
$$

As $\dot{\widehat{K}}_{1}=\dot{\widetilde{K}}_{1}, \dot{\widehat{K}}_{2}=\dot{\widetilde{K}}_{2}, \dot{\widehat{K}}_{3}=\dot{\widetilde{K}}_{3}$, and $\dot{\widehat{\delta}}_{a}=\dot{\widetilde{\delta}}_{a}$, substituting (27) and (28) into (30) yields

$$
\dot{V}=-e^{T} Q e \leq 0 .
$$

Since $V(t)$ is a positive definite function and $\dot{V}(t) \leq 0$, the signals $e(t), \widetilde{K}_{1}(t), \widetilde{K}_{2}(t), \widetilde{K}_{3}(t)$, and $\widetilde{\delta}_{a}(t)$ are all bounded. However, due to the modification of the reference model, the stability of the system cannot be directly proven. It is necessary to prove that one of the signals $x(t)$ or $x_{m}(t)$ is bounded.

Then, we define the following candidate Lyapunov function:

$$
W(x)=x^{T} P_{x} x
$$

Substituting (4) into (1) yields

$$
\begin{aligned}
\dot{x} & =\left(A+\delta_{a}\right) x+B_{v} K_{1}^{T}-B_{v} K_{1}^{T}+B_{u} \Delta u \\
& =\left(A_{m}+\delta_{a}\right) x-B_{v} K_{1}^{T}+B_{u} \Delta u .
\end{aligned}
$$

Based on (25), $A_{m}+\delta_{a}$ is assumed to satisfy the following Lyapunov inequality:

$$
\left(A_{m}+\delta_{a}\right)^{T} P_{x}+P_{x}\left(A_{m}+\delta_{a}\right)<-Q_{x},
$$

where $P_{x}=P_{x}^{T}, Q_{x}=Q_{x}^{T} \in \Re^{n \times n}$ are both positive definite matrices.

Differentiating (32), we can get

$$
\dot{W}(x)<-x^{T} Q_{x} x-2 x^{T} P_{x} B_{v} K_{1}^{T}+2 x^{T} P_{x} B_{u} \Delta u .
$$

Based on the previous results in [14], we know that there always exists a nonempty annulus region such that $\dot{W}(x)<$ 0 holds for all $x$ from that region. The boundedness of $x$ can be guaranteed as long as the initial conditions satisfy 
Theorem 4.1 in [14]. Since $x(t)$ is bounded and $x_{m}(t)=x(t)-$ $e(t)$, it is easy to conclude that $x_{m}(t)$ is bounded.

Consequently, according to the error dynamics in (26) and the boundedness of the closed-loop signals, we can get $\dot{e}(t) \in L_{\infty}$, and thus $\lim _{t \rightarrow \infty} e(t)=0$. Due to $e(t) \epsilon$ $L_{2} \cap L_{\infty}, \dot{\widehat{K}}_{1}, \dot{\widehat{K}}_{2}, \dot{\widehat{K}}_{3}, \dot{\widehat{\delta}}_{a} \in L_{2} \cap L_{\infty}$, and $x(t), r(t) \in L_{\infty}$, all signals and estimated parameters are bounded, realizing $\lim _{t \rightarrow \infty}\left[x(t)-x_{m}(t)\right]=0$.

The proof is completed.

Remark 8. Since the reference model is time varying, it is necessary to ensure that the parameter estimate $\widehat{\delta}_{a}$ remains within the stable region. If $\widehat{\delta}_{a}$ exceeds the stable region, a parameter projection method [18] will be utilized to ensure that the adaptive reference model has satisfactory stability and performance.

\section{Application Example}

To investigate the effectiveness of the proposed adaptive faulttolerant control scheme, we utilize a flight control example based on the ADMIRE model [19]. A low-speed flight case is considered, where the control surface efficiency is poor.

4.1. System Description. The state space of the model is given by (1), where the nominal values of the system matrices are in the appendix and

$$
\begin{aligned}
& x=\left[\begin{array}{llll}
\alpha & \beta & p & q
\end{array}\right]^{T}, \\
& y=\left[\begin{array}{lll}
\alpha & \beta
\end{array}\right]^{T}, \\
& u=\left[\begin{array}{llll}
\delta_{c} & \delta_{r e} & \delta_{l e} & \delta_{r}
\end{array}\right]^{T} .
\end{aligned}
$$

System states $\alpha, \beta, p, q$, and $r$ represent the angle of attack, the sideslip angle, the roll rate, the pitch rate, and the yaw rate, respectively. Control inputs $\delta_{c}, \delta_{r e}, \delta_{l e}$, and $\delta_{r}$ represent the positions of the canard wings, the right elevons, the left elevons, and the rudder, respectively.

The virtual control input $v$ contains the angular accelerations in roll, pitch, and yaw produced by the control surface.

The actuator position constraints are given by

$$
\begin{gathered}
\delta_{\min }=\left[\begin{array}{llll}
-25^{\circ} & -30^{\circ} & -30^{\circ} & -30^{\circ}
\end{array}\right]^{T}, \\
\delta_{\max }=\left[\begin{array}{llll}
25^{\circ} & 30^{\circ} & 30^{\circ} & 30^{\circ}
\end{array}\right]^{T} .
\end{gathered}
$$

It is assumed that the actuator $\delta_{c}$ loses $99 \%$ of effectiveness at $1.01 \mathrm{~s}$ while the remaining actuators are functional throughout and the aircraft experiences damage at $0 \mathrm{~s}$; therefore, a model mismatch exists in the system matrix from the outset. The model mismatch $\delta_{a}$ in the system is

$$
\delta_{a}=\left[\begin{array}{ccccc}
0 & 0 & 0 & 0.5 & 0 \\
0 & 0 & 0 & 0 & -0.5 \\
0 & 0 & 0 & 0 & 0 \\
0 & 0 & 0 & 0 & 0 \\
0 & 0 & 0 & 0 & 0
\end{array}\right]
$$

The reference inputs are given in Figure 2.
4.2. Controller Design. The matrices $K_{1}, K_{2}$ are designed using the linear quadratic regulator (LQR) method. The reference model is designed based on (4).

The quadratic stable region is obtained as $\delta_{a_{14}}$ and $\delta_{a_{25}} \epsilon$ $[-1.1564,1.1564]$. The security threshold is arbitrarily set at $80 \%$ of the maximum stable region. The corresponding Lyapunov matrix $P$ in (25) is obtained as

$$
P=\left[\begin{array}{ccccc}
0.0892 & 0.0568 & -0.007 & 0.0807 & 0.127 \\
0.0568 & 657.846 & 15.8863 & -0.1136 & -44.8585 \\
-0.007 & 15.8863 & 0.6474 & -0.0084 & -2.3445 \\
0.0807 & -0.1136 & -0.0084 & 0.1653 & 0.1152 \\
0.127 & -44.8585 & -2.3445 & 0.1152 & 47.7764
\end{array}\right] .
$$

Using (18), the matrix $K_{3}$ can be decided. The gains $\Gamma_{1}, \Gamma_{2}, \Gamma_{3}$, and $\Gamma_{a}$ are selected by trial and error.

The initial value of the weighting matrix $\widehat{W}$ is chosen as $\operatorname{diag}\{0.01,1,1,1\}$. Once actuator failures occur, the weighting matrix will be updated.

\subsection{Simulation Results}

Case 1. In the first case, all adaption is turned off; that is, the weighting matrix, the gains, and the parameter estimates are not updated. The plant states are shown in Figure 3. Obviously, the stability of the system cannot be guaranteed due to the presence of actuator failures, model mismatch, and input saturation.

Case 2. We utilize the adaptive fault-tolerant scheme in this case. The controller gain and parameter estimates are generated by the adaptive laws (27)-(28). At $t=1.01 \mathrm{~s}$, the first actuator fails while the remaining actuators continue to be functional, adaptively compensating for the failed first actuator. Unfortunately, actuators $\delta_{2}$ and $\delta_{4}$ have exceeded the input constraints as shown in Figure 4. Using the antisaturation algorithm, all of the actuators can be rigorously kept within the bounds, as shown in Figure 5. During the simulation, the parameter estimates $\widehat{\delta}_{a_{14}}$ and $\widehat{\delta}_{a_{25}}$ lie in the quadratic stable region throughout and approach the true value of model mismatch adaptively as shown in Figure 7. The plant with the adaptive reference model states and the tracking errors are illustrated in Figures 7 and 8, respectively. Simulation results show that the tracking errors remain small and converge to zero asymptotically.

\section{Conclusion}

This paper proposed a novel fault-tolerant control scheme for flight systems in the presence of simultaneous actuator failures, model mismatch, and input saturation. The scheme combined CA and MRAC to guarantee the system's stability and tracking performance. Practical conditions such as model mismatch and input saturation were taken into consideration in this paper. Due to model mismatch and input saturation, the original reference model might no longer be appropriate; therefore, we designed an adaptive reference model that also provides satisfactory performance. 


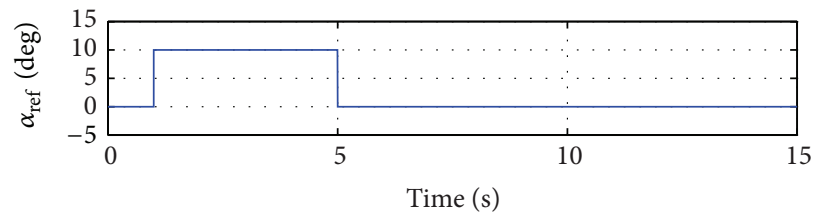

(a)

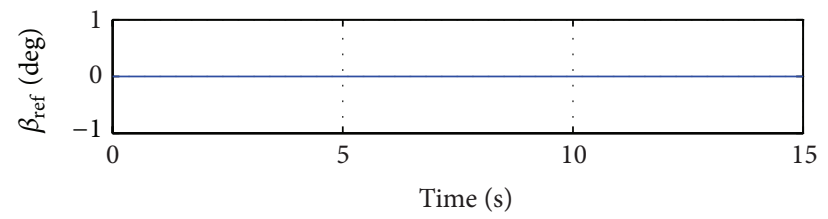

(b)

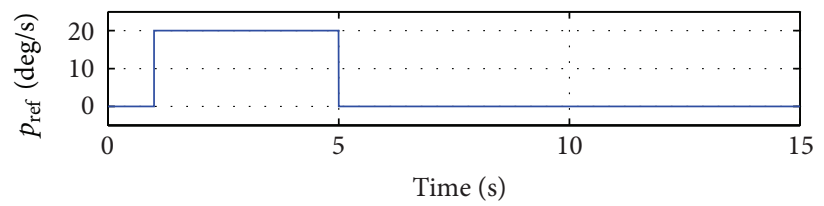

(c)

FIGURE 2: The reference inputs.

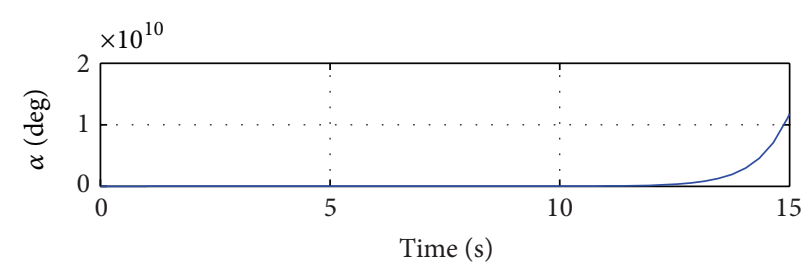

(a)

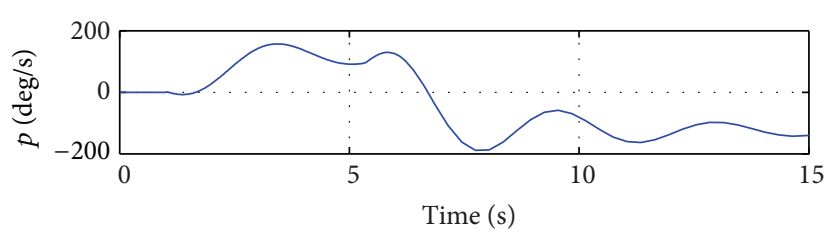

(c)

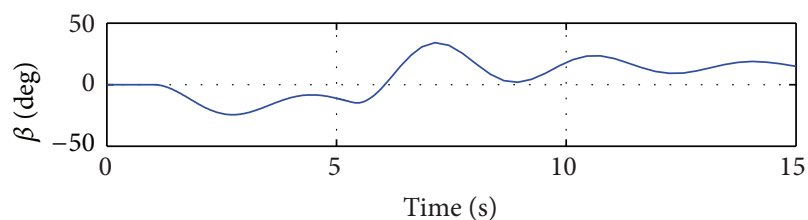

(b)

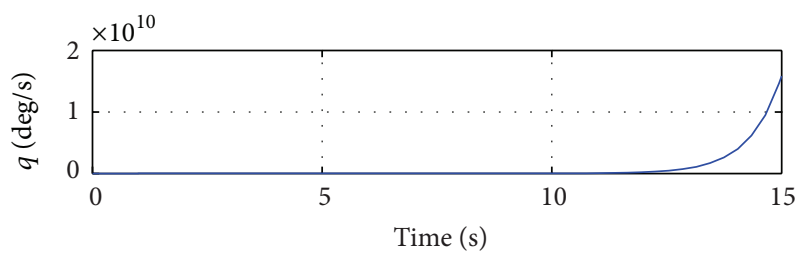

(d)

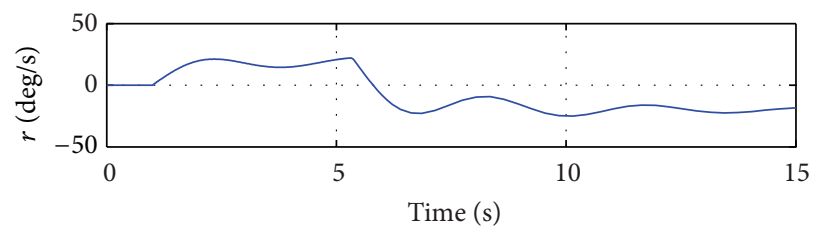

(e)

FIgUre 3: The system states of Case 1.

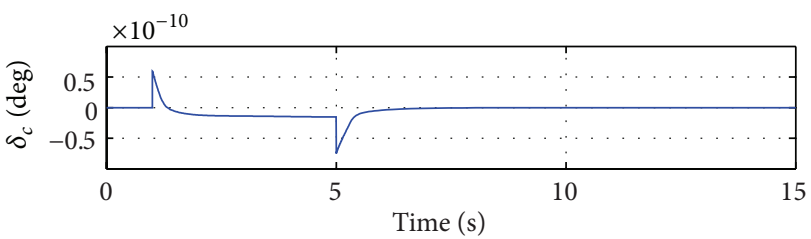

(a)

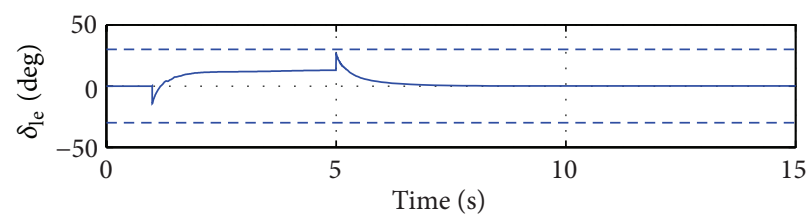

(c)

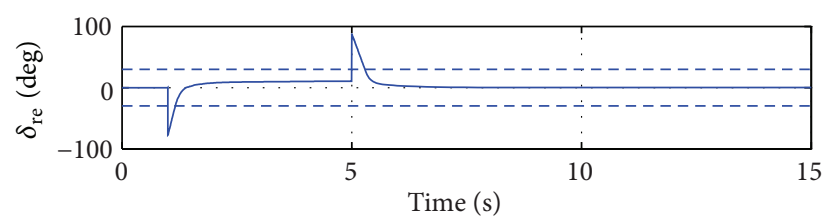

(b)

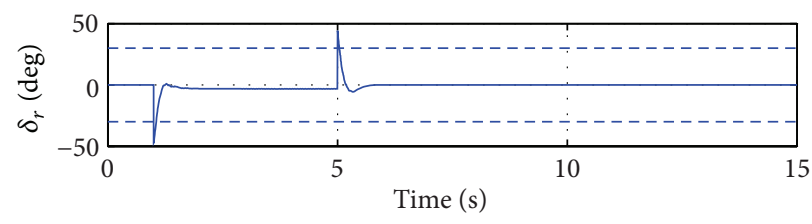

(d)

FIgURE 4: The adaptive control input $u_{a}$ of Case 2. 


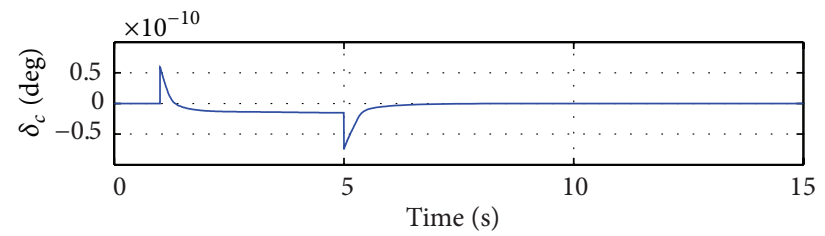

(a)

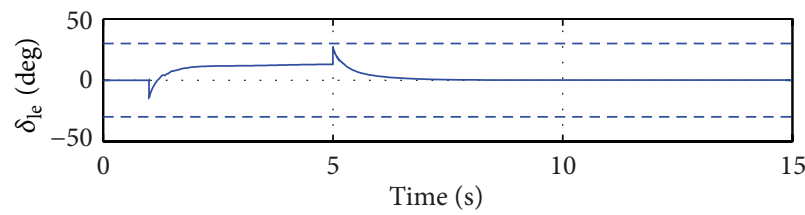

(c)

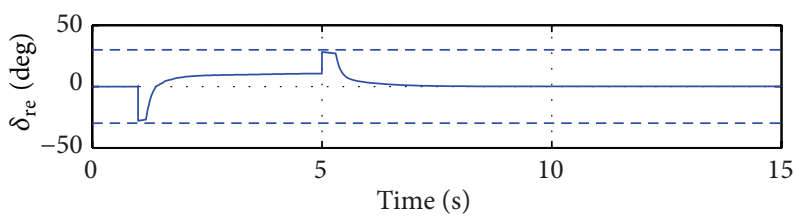

(b)

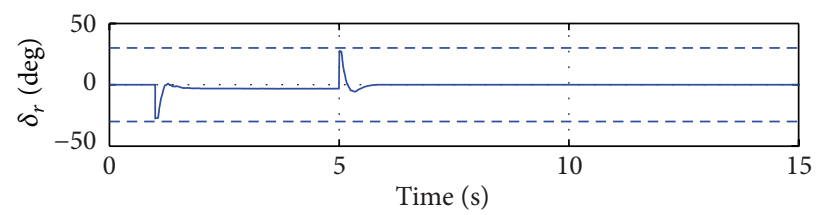

(d)

Figure 5: The commanded control input $u_{c}$ of Case 2 .

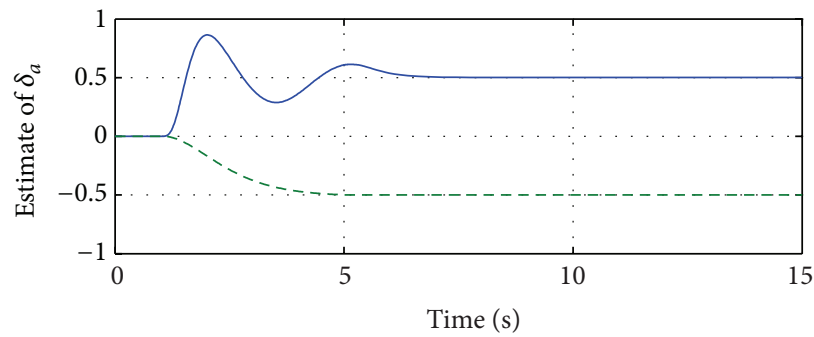

$-\delta_{14}$

Figure 6: The model mismatch estimate of Case 2.

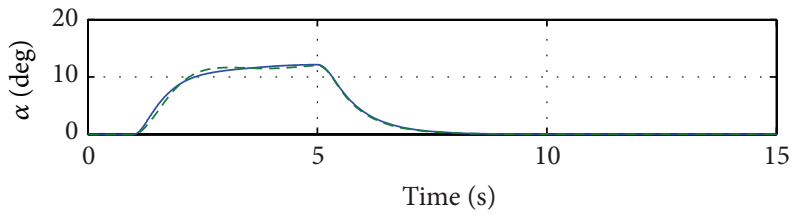

(a)

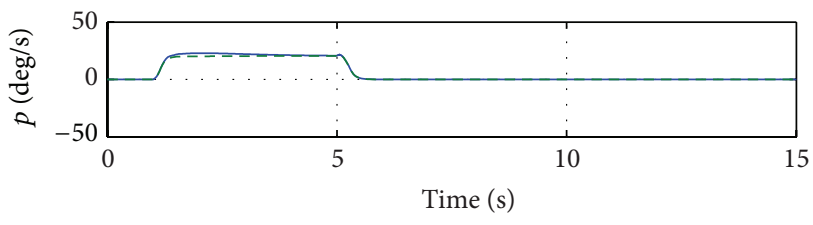

(c)

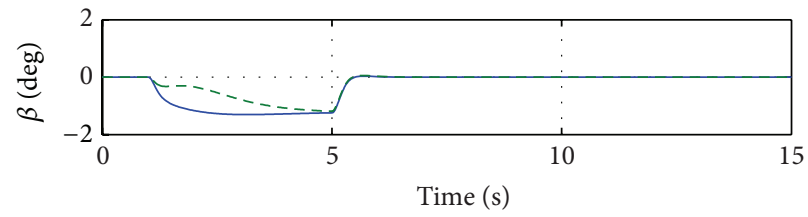

(b)

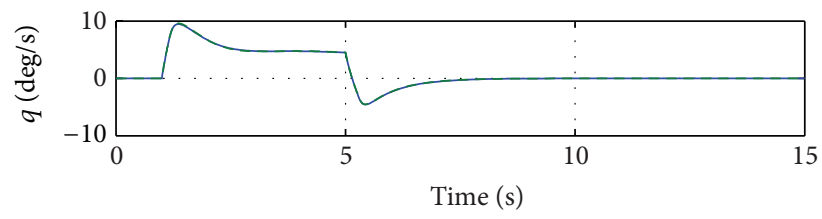

(d)

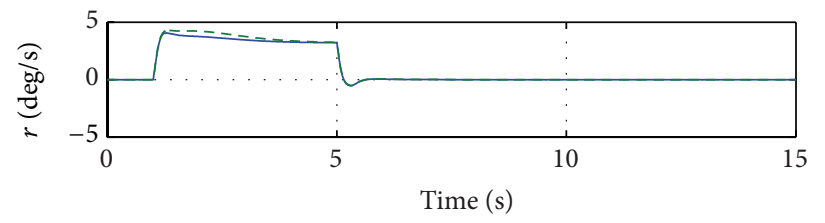

$-x$

$---x_{m}$

(e)

Figure 7: The system states of Case 2. 


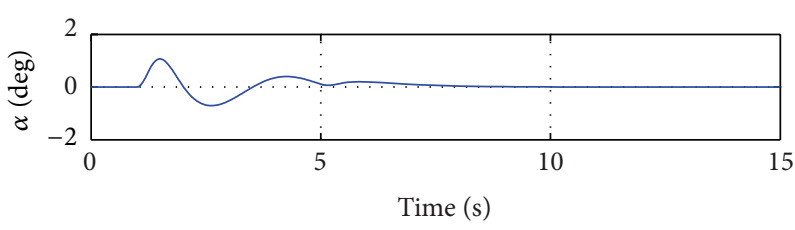

(a)

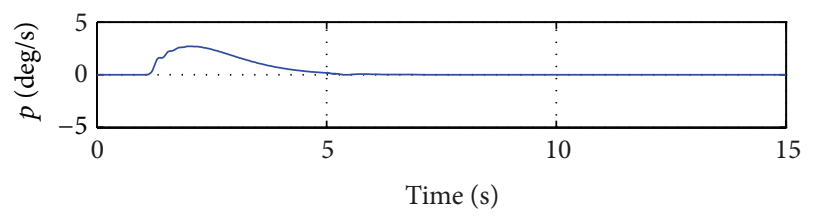

(c)

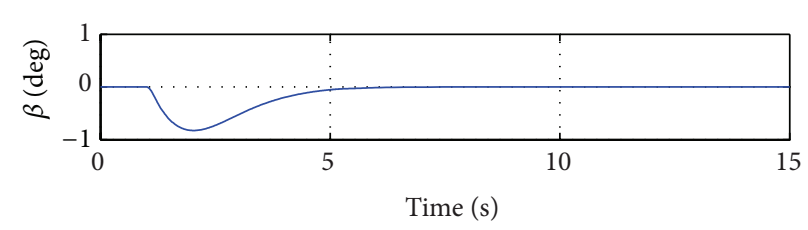

(b)

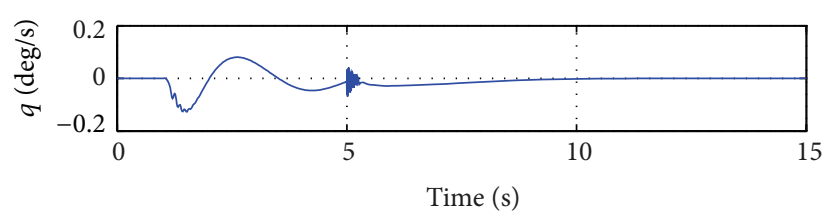

(d)

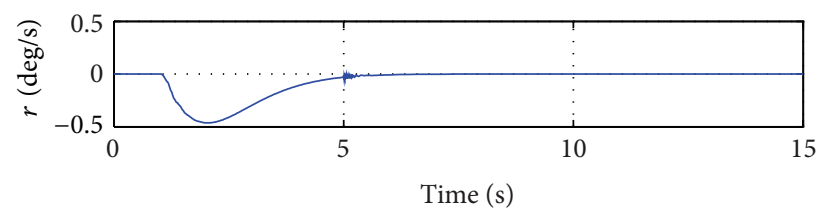

(e)

FIGURE 8: The tracking errors of Case 2.

Simulation results indicated that mismatch estimation, input compensation, and satisfactory tracking performance could be obtained in the presence of actuator failures, model mismatch, and input saturation. Future investigation will be necessary to achieve adaptive fault estimation and test more complex and realistic models. The FTC of multiple flight systems will be explored in our future study.

\section{Appendix}

Consider the following:

$$
\begin{gathered}
A=\left[\begin{array}{ccccc}
-0.5432 & 0.0137 & 0 & 0.9778 & 0 \\
0 & -0.1179 & 0.2215 & 0 & -0.9661 \\
0 & -10.5128 & -0.9967 & 0 & 0.6176 \\
2.6221 & -0.0030 & 0 & -0.5057 & 0 \\
0 & 0.7075 & -0.0939 & 0 & -0.2127
\end{array}\right], \\
C=\left[\begin{array}{lllll}
1 & 0 & 0 & 0 & 0 \\
0 & 1 & 0 & 0 & 0 \\
0 & 0 & 1 & 0 & 0
\end{array}\right], \\
B=\left[\begin{array}{cccc}
0 & -4.2423 & 4.2423 & 1.4871 \\
1.6532 & -1.2735 & -1.2735 & 0.0024 \\
0 & -0.2805 & 0.2805 & -0.8823
\end{array}\right] \\
B_{v}=\left[\begin{array}{l}
0_{2 \times 3} \\
I_{3 \times 3}
\end{array}\right], \quad B_{u}=B_{v} B .
\end{gathered}
$$

\section{Conflict of Interests}

The authors declare that there is no conflict of interests regarding the publication of this paper.

\section{Acknowledgments}

This work is supported by the National Natural Science Key Foundation of China under Grant no. 91216124, no. 91116002, no. 90916003 , and no. 91216304 , the foundation of National Astronautica Key Lab (2012-865-6) and Aeronautical Science Foundation of China under Grant no. 20100171001.

\section{References}

[1] H. Alwi and C. Edwards, "Fault tolerant control using sliding modes with on-line control allocation," Automatica, vol. 44, no. 7, pp. 1859-1866, 2008.

[2] M. T. Hamayun, C. Edwards, and H. Alwi, "Design and analysis of an integral sliding mode fault-tolerant control scheme," IEEE Transactions on Automatic Control, vol. 57, no. 7, pp. 1783-1789, 2012.

[3] D. Kim and Y. Kim, "Robust variable structure controller design for fault tolerant flight control," Journal of Guidance, Control, and Dynamics, vol. 23, no. 3, pp. 430-437, 2000.

[4] W. Chen and J. Jiang, "Fault-tolerant control against stuck actuator faults," IEE Proceedings Control Theory and Applications, vol. 152, no. 2, pp. 138-146, 2005.

[5] G. Tao, S. M. Joshi, and X. Ma, "Adaptive state feedback and tracking control of systems with actuator failures," IEEE Transactions on Automatic Control, vol. 46, no. 1, pp. 78-95, 2001.

[6] L. Cui and Y. Yang, "Disturbance rejection and robust leastsquares control allocation in flight control system," Journal of Guidance, Control, and Dynamics, vol. 34, no. 6, pp. 1632-1643, 2011.

[7] D. Enns, "Control allocation approaches," in Proceedings of the AIAA Guidance, Navigation, and Control Conference, pp. 98108, 1998. 
[8] O. Härkegård and S. T. Glad, "Resolving actuator redundancyoptimal control vs. control allocation," Automatica, vol. 41, no. 1, pp. 137-144, 2005.

[9] W. C. A. Kishore, S. Sen, and G. Ray, "Disturbance rejection and control allocation of over-actuated systems," in Proceedings of the IEEE International Conference on Industrial Technology (ICIT '06), pp. 1054-1059, December 2006.

[10] K. A. Bordignon and W. C. Durham, "Closed-form solutions to constrained control allocation problem," Journal of Guidance, Control, and Dynamics, vol. 18, no. 5, pp. 1000-1007, 1995.

[11] J. D. Bošković and R. K. Mehra, "Control allocation in overactuated aircraft under position and rate limiting," in Proceedings of the American Control Conference, pp. 791-796, May 2002.

[12] S. M. Joshi, G. Taoy, and T. Khong, "A direct adaptive control approach in the presence of model mismatch," in Proceedings of the AIAA Guidance, Navigation, and Control Conference and Exhibit, pp. 10-13, August 2009.

[13] S. M. Joshi, G. Tao, and P. Patre, "Direct adaptive control using an adaptive reference model," International Journal of Control, vol. 84, no. 1, pp. 180-196, 2011.

[14] A. Ajami, Adaptive flight control in the presence of input constraints [M.S. thesis], Aerospace Engineering Department, Virginia Polytechnic Institute and State University, Blacksburg, Va, USA, 2005.

[15] G. Wen, Z. Duan, Z. Li, and G. Chen, "Consensus and its $L_{2}$-gain performance of multi-agent systems with intermittent information transmissions," International Journal of Control, vol. 85, no. 4, pp. 384-396, 2012.

[16] G. Wen, Z. Duan, W. Yu, and G. Chen, "Consensus of multiagent systems with nonlinear dynamics and sampled-data information: a delayed-input approach," International Journal of Robust and Nonlinear Control, vol. 23, no. 6, pp. 602-619, 2013.

[17] G. Wen, Z. Duan, W. Ren, and G. Chen, "Distributed consensus of multi-agent systems with general linear node dynamics and intermittent communications," International Journal of Robust and Nonlinear Control.

[18] S. M. Joshi, P. Patre, and G. Tao, "Adaptive control of systems with actuator failures using an adaptive reference model," Journal of Guidance, Control, and Dynamics, vol. 35, no. 3, pp. 938-949, 2012.

[19] O. Härkegård, Backstepping and control allocation with applications to flight control [Ph.D. thesis], Electrical Engineering Department, Linköping University, Linköping, Sweden, 2003. 


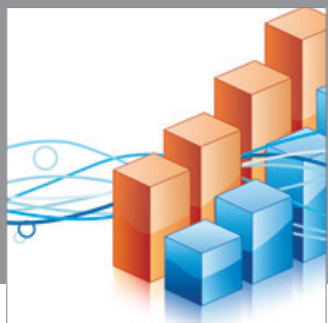

Advances in

Operations Research

mansans

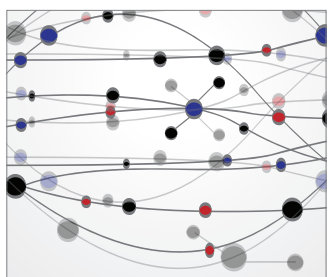

The Scientific World Journal
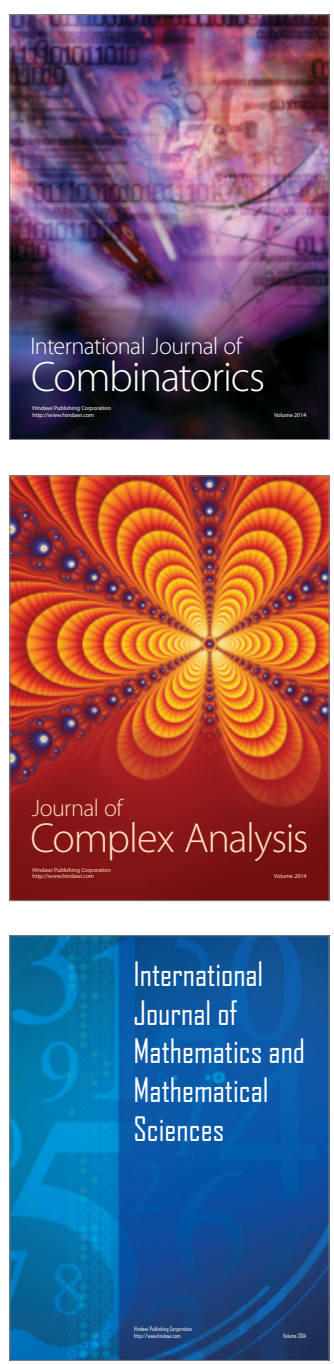
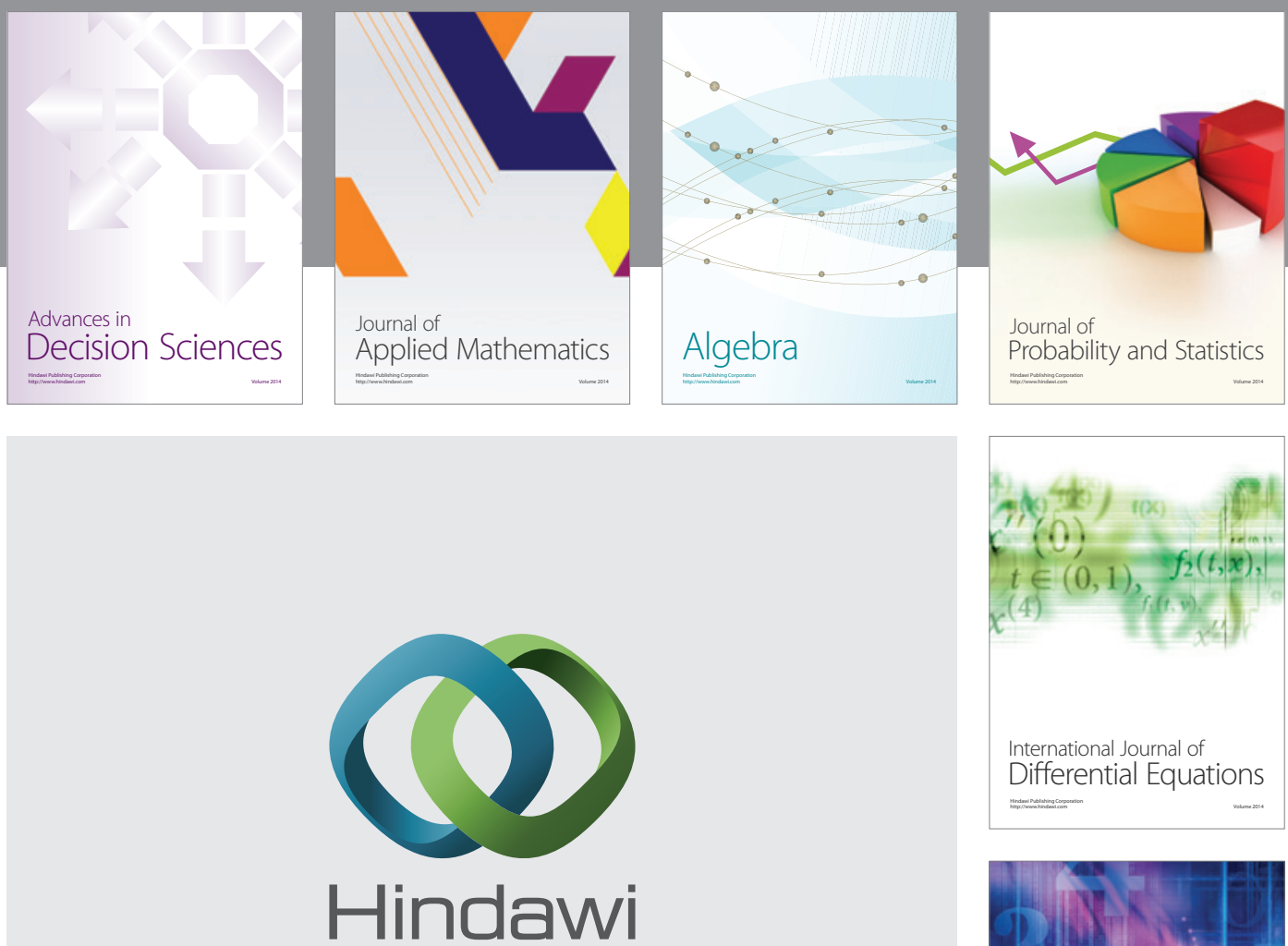

Submit your manuscripts at http://www.hindawi.com
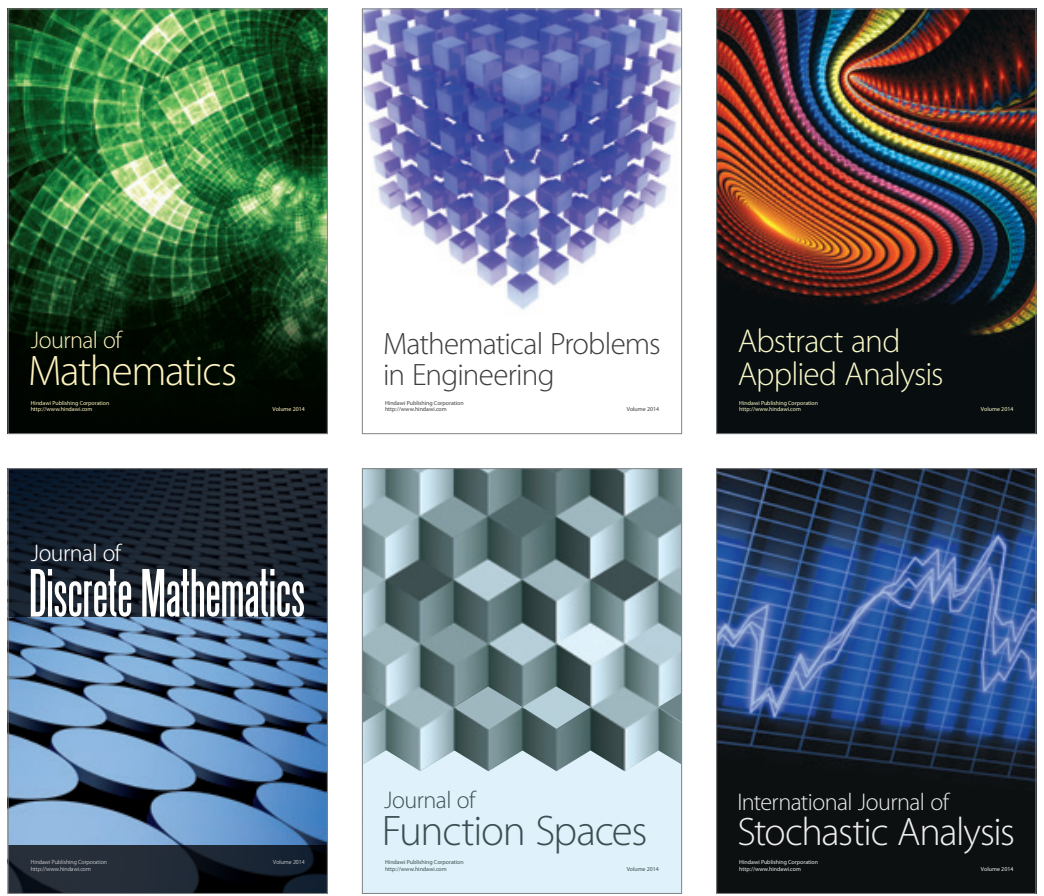

Journal of

Function Spaces

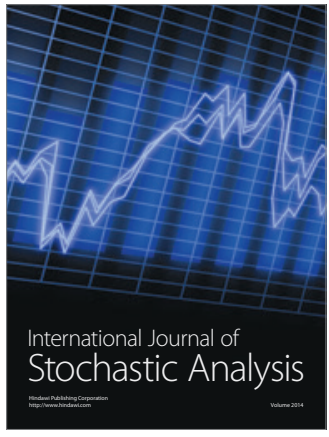

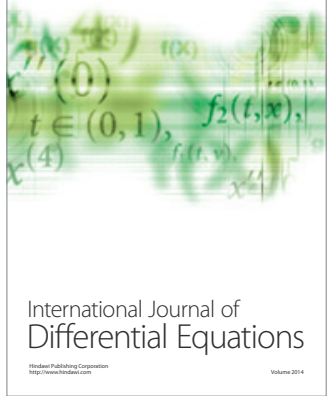
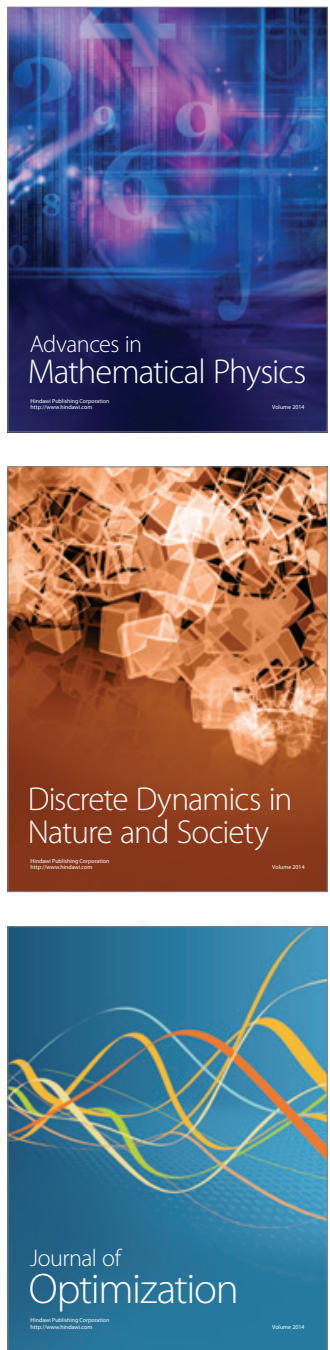